\title{
Have biomarkers failed in acute kidney injury? We are not sure
}

\author{
John R. Prowle ${ }^{1}$ and Mitchell H. Rosner ${ }^{2}$ \\ ${ }^{1}$ Queen Mary University of London Adult Critical Care Unit Royal London Hospital \\ Whitechapel Road London E1 1BB \\ ${ }^{2}$ Division of Nephrology, University of Virginia Health System, Charlottesville, Virginia 22908
}

Over the past decade, there has been an explosion of interest in the study of urinary and plasma biomarkers for the rapid diagnosis, molecular phenotyping and prognostication of acute kidney injury (AKI) with over 3300 publications devoted to this topic listed in PubMed during the past 10 years. More than 15 separate biomarkers plasma and urine have been identified and investigated to various levels, the majority in the context of AKI complicating critical illness[1]. However, opinion remains divided as to the clinical value of biomarker measurements in the care of patients at risk or with acute kidney injury as reflected in opposing opinion pieces in this issue of Intensive Care Medicine. While we agree that AKI biomarkers are not ready for implementation in routine care, we feel that progress is being made and that ultimately there may be important roles for these tools in the care of critically ill patients.

The ultimate goal of biomarkers should be to improve outcomes of patients with AKI and thus far, this high benchmark has not been realized. Much of the early promise of biomarkers was for rapid diagnosis of AKI at the earliest time-period after injury. The supposition is that early diagnosis would facilitate avoidance of further injurious exposures as well as allow administration of therapies that might have been limited because we had relied on late markers of AKI (such as creatinine) which are manifest after significant and irreversible injury has already occurred. Studies of biomarkers have occurred in several large prospective cohorts with differing clinical settings (post-surgery, post-procedure, sepsis, emergency department) and different timing of biomarker measurements. However, in just about every study, the discrimination for AKI detection is modest with area under the curves (AUCs) 
typically between 0.7 and 0.8 for most biomarkers[1]. Notably in the circumstances where biomarkers performed best, such as pediatric cardiac surgery[2], and in animal models[3], the renal insult was precisely timed, the cause of AKI fairly-uniform and the clinical population homogenous. However, it is in the chaotic environment of critically ill adults, with comorbid disease and multiple insults of uncertain timing where diagnostics for AKI are needed. However, in these settings, the results have been mixed and disappointing. This should not be surprising; $\mathrm{AKI}$ is not a single disease with one pathogenic pathway, but a heterogeneous clinical syndrome of mixed causes with variable underlying pathology and clinical course. Furthermore, we currently diagnose the most common underlying pathology of AKI, renal tubular injury, based on changes in serum creatinine, a glomerular-filtration marker that may be highly confounded by the effects of acute critical illness and its treatment. Finally, AKI is a dynamic process that will be modified by host response to illness, medical interventions and complicating conditions and there is a point when 'early diagnosis' turns into to 'predicting the future'. Thus, asking a biomarker to accurately predict an evolving clinical process against a highly confounded diagnostic standard may be an impossible task as currently defined[4].

Alternatively, the diverse field of AKI biomarkers could provide important potential insights into the pathophysiology and mechanisms of $\mathrm{AKI}$, such as renal inflammatory responses, cellular responses to injury (such as cell cycle arrest) and tubular vs. glomerular function. Importantly the underlying cellular and molecular processes in AKI may differ considerably despite a similar biochemical profile of renal dysfunction[5]. These are important biological insights that get lost when biomarkers are used merely as a diagnostic tool and most importantly they may point to effective therapeutics (pharmaco-diagnostic testing). As shown in figure 1, biomarkers may be useful at several stages in the care of patient: the rapid diagnosis of $\mathrm{AKI}$, determining the type and site of injury and in establishing the prognosis of AKI both in the short and long-run. However, to be able to use biomarkers appropriately we must be able to integrate this information into our clinical practice and decision making.

So where does this leave us in 2017 and moving forward? We believe we must escape the singular mindset that we are going to use renal biomarkers to 'diagnose AKI' as we currently 
define it, particularly as we have no proven AKI-targeted interventions to offer apart from supportive care. However, to discount the value of biomarkers in the care of patients with AKI would be a mistake. For example, the positive predictive values for identification of AKI are poor (10-30\%), but importantly, negative predictive values are good (86-97\%)[6]. Thus, clinical value may lie in directing of low-risk patients away from expensive interventions (such as ICU care), much in the way that d-dimer, troponin and BNP are best used to rule out venous thromboembolism, myocardial infarction and heart failure, respectively.

It is critical to understand that changes in biomarker levels describe a real biological process and this may allow clinicians to think about AKI in very different ways. The days of equating AKI with acute tubular necrosis may be coming to a close and instead we may use biomarkers to phenotype injuries and pathological processes with an eventual goal of developing more effective therapies. For example, IL-18 is an attractive target for biomarkerdirected therapy of $\mathrm{AKI}$, because this cytokine seems to play a prominent role in the inflammatory processes that exacerbate renal injury during the extension phase of AKI[7]. Therapies that disrupt the IL-18-signaling axis itself could prove efficacious, because work done in animal models of AKI has consistently shown that doing so attenuates renal injury[7].

We would also argue that it is time to develop more global strategies in the care of patients with AKI. These strategies include thinking about several meaningful clinical outcomes such as survival, length of stay or long term renal function, rather than any conventional AKI criteria. However, such an approach requires the correct combination of the biomarker, setting and intervention.

The promise of biomarkers is perhaps shown in a recent single center study that described the use of a urinary TIMP2xIGFBP7[8] to target a bundle of AKI-preventative care to patients after cardiac surgery, with evidence of reduced incidence of AKI in biomarker positive patients randomized to the intervention, but without signal of benefit in terms of use of RRT and long-term renal function. This last result is encouraging in that it demonstrates potential benefit of a biomarker to identify a high-risk group for a preventative intervention; however 
interpretation of the results remains limited by the use of urine and creatinine defined-AKI as an imperfect surrogate endpoint.

In summary, on the one hand we are expecting far too much of AKI biomarkers - that they will universally be able to precisely predict the development of significant AKI of all forms - and on the other expecting far too little - neglecting unique insights into underlying renal pathology that could be targets for therapy? Our premise is that while biomarkers are not ready for primetime use and future studies are clearly required, some applications such as identification of low-risk patients for lower-intensity care can be easily tested for safety and cost-effectiveness. Conversely, interventional studies of biomarker targeted intervention are a very promising approach avoiding the limitations of conventional AKI diagnosis, but require careful matching of biomarker, setting and intervention to be successful.

\section{References}

1. Malhotra R, Siew ED, (2017) Biomarkers for the Early Detection and Prognosis of Acute Kidney Injury. Clin J Am Soc Nephrol 12: 149-173

2. Mishra J, Dent C, Tarabishi R, Mitsnefes MM, Ma Q, Kelly C, Ruff SM, Zahedi K, Shao M, Bean J, Mori K, Barasch J, Devarajan P, (2005) Neutrophil gelatinase-associated lipocalin (NGAL) as a biomarker for acute renal injury after cardiac surgery. Lancet 365: 1231-1238

3. Paragas N, Qiu A, Zhang Q, Samstein B, Deng SX, Schmidt-Ott KM, Viltard M, Yu W, Forster CS, Gong G, Liu Y, Kulkarni R, Mori K, Kalandadze A, Ratner AJ, Devarajan P, Landry DW, D'Agati V, Lin CS, Barasch J, (2011) The Ngal reporter mouse detects the response of the kidney to injury in real time. Nat Med 17: 216-222

4. Waikar SS, Betensky RA, Emerson SC, Bonventre JV, (2012) Imperfect gold standards for kidney injury biomarker evaluation. J Am Soc Nephrol 23: 13-21

5. Xu K, Rosenstiel P, Paragas N, Hinze C, Gao X, Huai Shen T, Werth M, Forster C, Deng R, Bruck E, Boles RW, Tornato A, Gopal T, Jones M, Konig J, Stauber J, D'Agati V, Erdjument-Bromage H, Saggi S, Wagener G, Schmidt-Ott KM, Tatonetti N, Tempst P, Oliver JA, Guarnieri P, Barasch J, (2016) Unique Transcriptional Programs Identify Subtypes of AKI. J Am Soc Nephrol

6. Alge JL, Arthur JM, (2015) Biomarkers of AKI: a review of mechanistic relevance and potential therapeutic implications. Clin J Am Soc Nephrol 10: 147-155

7. Wu H, Craft ML, Wang P, Wyburn KR, Chen G, Ma J, Hambly B, Chadban SJ, (2008) IL-18 contributes to renal damage after ischemia-reperfusion. J Am Soc Nephrol 19: 2331-2341

8. Meersch M, Schmidt C, Hoffmeier A, Van Aken H, Wempe C, Gerss J, Zarbock A, (2017) Prevention of cardiac surgery-associated AKI by implementing the KDIGO guidelines in high risk patients identified by biomarkers: the PrevAKI randomized controlled trial. Intensive care medicine 


\section{Figure 1. The Promise of Biomarkers}

\section{The Promise of Biomarkers}

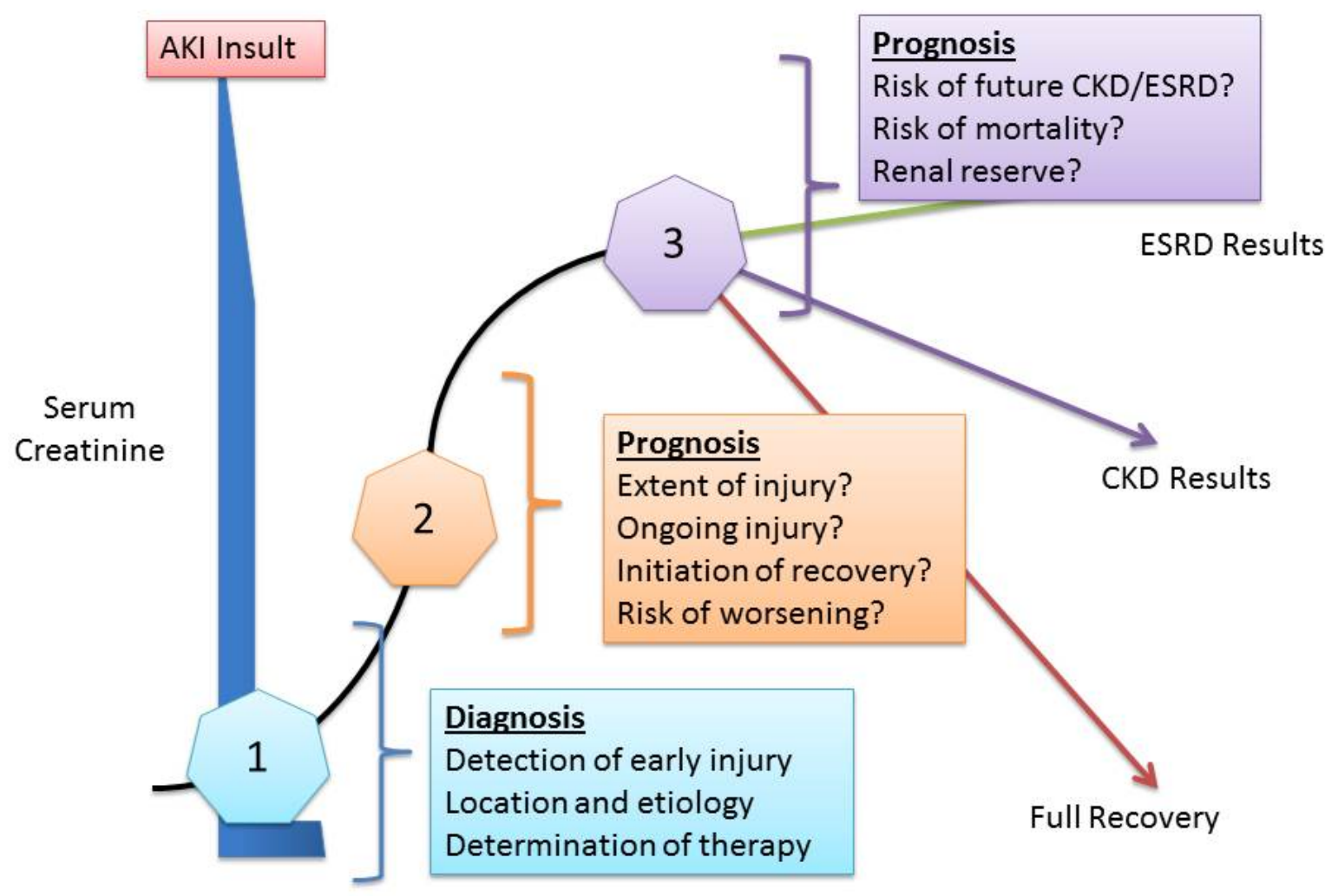

The course of AKI begins at the initial injury (point 1) where biomarkers may be useful in early diagnosis, determination of the location of injury and possibly in molecular phenotyping which could lead to specific therapies. As AKI evolves (points 2 and 3), biomarkers may be useful in prognostication and help clinicians determine whether there is ongoing injury with a risk of worsening kidney function or if there are signs of recovery. Biomarkers may also provide information on the future risk of chronic kidney disease (CKD), end-stage renal disease (ESRD) or mortality. 